Article

\title{
Evidence of Accelerated Evolution and Ectodermal-Specific Expression of Presumptive BDS Toxin cDNAs from Anemonia viridis
}

\author{
Aldo Nicosia ${ }^{1}$, Teresa Maggio ${ }^{2}$, Salvatore Mazzola ${ }^{3}$ and Angela Cuttitta ${ }^{1, *}$ \\ 1 Laboratory of Molecular Ecology and Biotechnology, National Research Council, Institute for \\ Marine and Coastal Environment (IAMC-CNR), Detached Unit of Capo Granitola, \\ Torretta Granitola 91021, Trapani, Italy; E-Mail: aldo.nicosia@iamc.cnr.it \\ 2 Institute for Environmental Protection and Research-ISPRA, Palermo 90143, Italy; \\ E-Mail: teresa.maggio@isprambiente.it \\ 3 National Research Council, Institute for Marine and Coastal Environment (IAMC-CNR), \\ Calata Porta di Massa, Napoli 80133, Italy; E-Mail: salvatore.mazzola@cnr.it \\ * Author to whom correspondence should be addressed; E-Mail: angela.cuttitta@iamc.cnr.it; \\ Tel.: +39-0924-40600; Fax: +39-0924-40445.
}

Received: 21 August 2013; in revised form: 10 September 2013 / Accepted: 13 September 2013 / Published: 30 October 2013

\begin{abstract}
Anemonia viridis is a widespread and extensively studied Mediterranean species of sea anemone from which a large number of polypeptide toxins, such as blood depressing substances (BDS) peptides, have been isolated. The first members of this class, BDS-1 and BDS-2, are polypeptides belonging to the $\beta$-defensin fold family and were initially described for their antihypertensive and antiviral activities. BDS-1 and BDS-2 are 43 amino acid peptides characterised by three disulfide bonds that act as neurotoxins affecting Kv3.1, Kv3.2 and Kv3.4 channel gating kinetics. In addition, BDS-1 inactivates the Nav1.7 and Nav1.3 channels. The development of a large dataset of $A$. viridis expressed sequence tags (ESTs) and the identification of 13 putative BDS-like cDNA sequences has attracted interest, especially as scientific and diagnostic tools. A comparison of BDS cDNA sequences showed that the untranslated regions are more conserved than the protein-coding regions. Moreover, the $K_{\mathrm{A}} / K_{\mathrm{S}}$ ratios calculated for all pairwise comparisons showed values greater than 1, suggesting mechanisms of accelerated evolution. The structures of the BDS homologs were predicted by molecular modelling. All toxins possess similar 3D structures that consist of a triple-stranded antiparallel $\beta$-sheet and an additional small antiparallel $\beta$-sheet located downstream of the cleavage/maturation site; however, the orientation of the
\end{abstract}


triple-stranded $\beta$-sheet appears to differ among the toxins. To characterise the spatial expression profile of the putative BDS cDNA sequences, tissue-specific cDNA libraries, enriched for BDS transcripts, were constructed. In addition, the proper amplification of ectodermal or endodermal markers ensured the tissue specificity of each library. Sequencing randomly selected clones from each library revealed ectodermal-specific expression of ten BDS transcripts, while transcripts of BDS-8, BDS-13, BDS-14 and BDS-15 failed to be retrieved, likely due to under-representation in our cDNA libraries. The calculation of the relative abundance of BDS transcripts in the cDNA libraries revealed that BDS-1, BDS-3, BDS-4, BDS-5 and BDS-6 are the most represented transcripts.

Keywords: Anemonia viridis; BDS peptides; accelerated evolution; molecular modelling; tissue-specific libraries; gene expression pattern

\section{Introduction}

Sea anemones are generally poisonous animals that spend most of their lives in a sessile form; hence, capturing activities and defence mechanisms are strongly associated with toxin production [1]. The sea anemone Anemonia viridis is a widespread and extensively studied Mediterranean species [2-6], from which a large number of polypeptide toxins, including sodium and potassium ion channel modulators or blockers, as well as Kunitz-type protease inhibitors, have been isolated [7].

The $\mathrm{K}^{+}$channel-blocking toxins can be grouped into different classes (type 1-4) based on the number of amino acid residues, molecular structure and target [8,9]. BDS peptides belong to the type 3 class, which includes peptides of various origins, such as APETx1 and APETx2 from Anthopleura elegantissima [10] or Am-II from Antheopsis maculata [11]. The first members of this class, BDS-1 and -2, were isolated 15 years ago and were originally described as blood pressure-reducing substances with antiviral activities [12]. BDS-1 and BDS-2 are 43 amino acid, cysteine-rich polypeptides characterised by three disulfide bonds. The peptides act on $\mathrm{K}^{+}$channels containing Kv3 subunits, such as Kv3.1, 3.2, and 3.4; however, these peptides do not block Kv1.2, 1.3, $1.4,1.5,2.2,4.2$ or $4.3 \mathrm{~K}^{+}$channels [13,14]. Ion channel inhibition develops as a result of the modification of Kv3 gating kinetics and not by the direct blockage of the channel pore [15]. Although BDS toxins were previously considered ineffective on sodium channels, BDS-1 was recently shown to strongly inactivate Nav1.7 channels and weakly inhibit Nav1.3 channels [16]. In addition, BDS-1 was also found to prevent neuronal death mediated by $\beta$-amyloid peptide through inhibition of Kv3.4-generated current [17].

Recently, the development of high-throughput sequencing technologies associated with digital transcriptome platforms has allowed the discoveries of novel members of known classes of toxins as well as novel peptide structures previously unknown in cold water sea anemones, such as Bolocera tuediae and Hormathia digitata [18].

Because toxins from natural venoms are a source of considerable scientific and diagnostic tools, the development of a large dataset of A. viridis ESTs [19] has attracted much interest. A search for 
toxin-encoding transcripts using a single residue distribution analysis (SRDA) in the EST database retrieved 12 putative BDS-like cDNA sequences (BDS-3 to BDS-14) in addition to BDS-1 [20].

In the present work, an open reading frame (ORF) for an additional putative BDS homolog (BDS-15) was recovered from the A. viridis EST collection, and in silico studies were performed on the peptide homologs constituting the arsenal of BDS toxins.

A comparison of BDS cDNA sequences showed that the untranslated regions (UTR) are more conserved than the protein-coding regions, which suggests mechanisms for accelerated evolution. A phylogenetic analysis and protein homology modelling were used to investigate the evolutionary relationships among the toxins affecting $\mathrm{K}^{+}$channels and to establish conserved structural motifs. Furthermore, the efficient separation of the ectoderm from the endodermal layers of sea anemones enabled us to construct BDS tissue-specific cDNA libraries. Tissue expression profiles of the BDS toxins were analysed, and an ectodermal-restricted expression pattern emerged.

\section{Results and Discussion}

\subsection{A. viridis $B D S c D N A$ Characterisation}

The cDNAs encoding the BDS toxins described previously [20] and BDS-15 were found in the EST database of the sea anemone $A$. viridis. A multiple sequence alignment was constructed to compare cDNAs encoding BDS peptides (Figure 1) and accession numbers for the ESTs are provided in Table 1. The open reading frames (ORFs) of the BDSs ranged from 231 to $249 \mathrm{bp}$, which encode proteins of 76 to 82 amino acids (Table 1). The stop codon was usually TAG, except for BDS-10, where it was replaced by TGA.

Figure 1. Multiple sequence alignment of BDS cDNAs from $A$. viridis. The cDNAs were aligned using ClustalW2. Dashes indicate gaps introduced to maximise the alignment. The ATG start codon is indicated by a purple box, while in-frame stop codons (TAG or TGA) are boxed in blue. The numbering of nucleotides refers to the alignment.

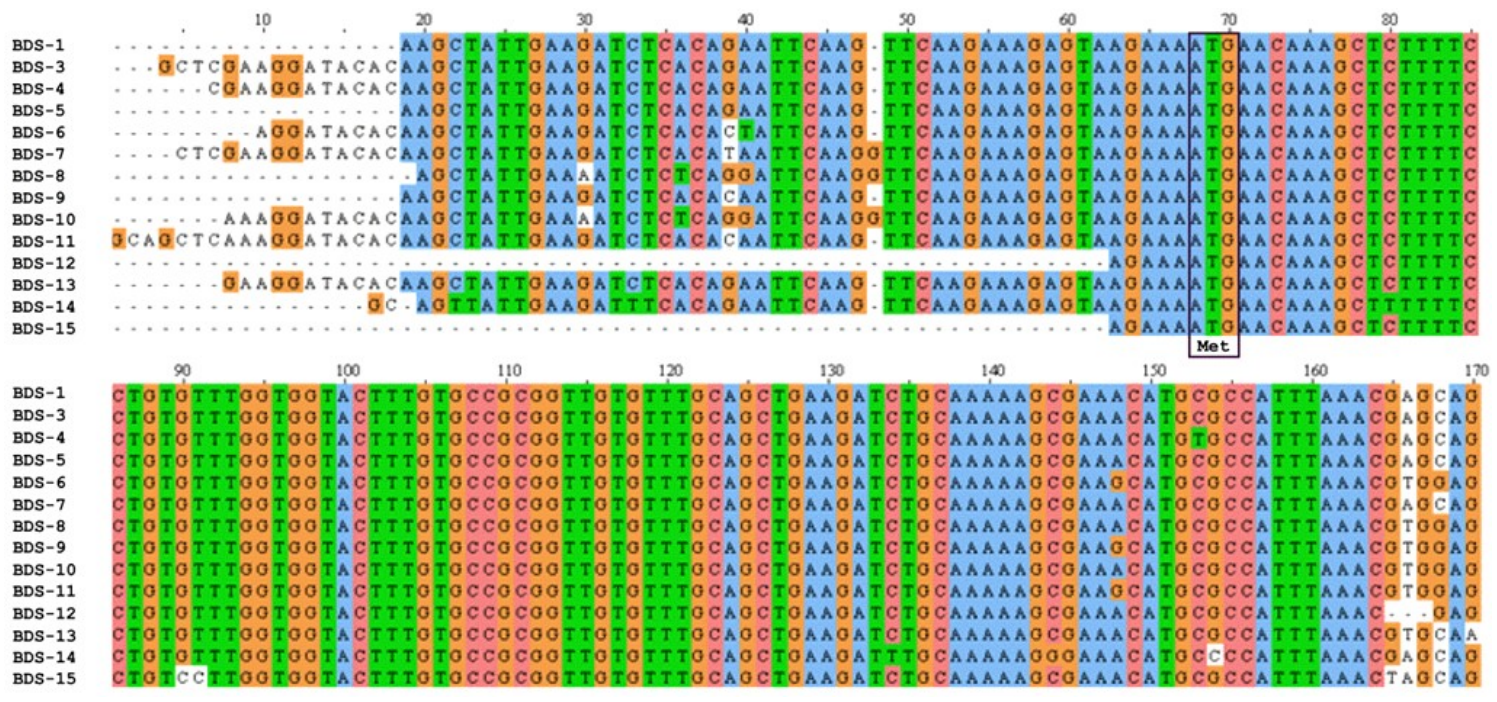


Figure 1. Cont.

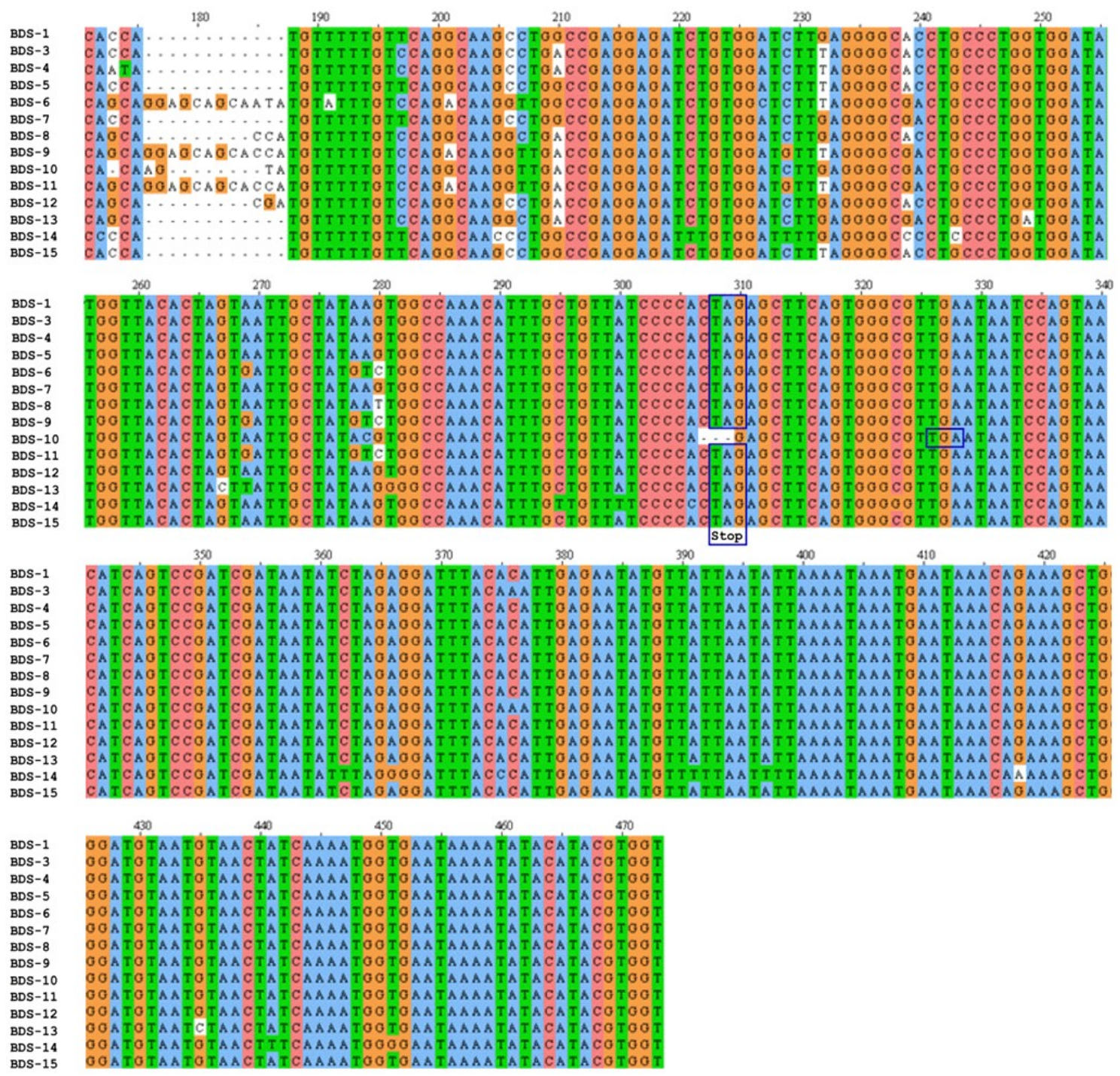

Table 1. BDS peptides in A. viridis.

\begin{tabular}{cccccc}
\hline Accession Number & Toxin & ORF length $^{\mathbf{a}}$ & Protein length $^{\mathbf{b}}$ & Molecular weight $^{\mathbf{c}}$ & $\mathbf{p I}^{\mathbf{d}}$ \\
\hline FK728690 & BDS-1 & 231 & 76 & 8342.8 & 9.02 \\
FK744472 & BDS-3 & 231 & 76 & 8444.9 & 8.86 \\
FK722457 & BDS-4 & 231 & 76 & 8489.0 & 8.86 \\
FK720902 & BDS-5 & 231 & 76 & 8376.9 & 9.02 \\
FK754940 & BDS-6 & 243 & 80 & 8671.2 & 8.14 \\
FK736435 & BDS-7 & 231 & 76 & 8356.8 & 8.86 \\
FK723172 & BDS-8 & 234 & 77 & 8427.9 & 8.68 \\
FK725608 & BDS-10 & 249 & 82 & 9041.5 & 8.68 \\
FK740326 & BDS-11 & 243 & 80 & 8765.2 & 7.52 \\
FK736010 & BDS-12 & 231 & 76 & 8470.0 & 9.02 \\
FK752236 & BDS-13 & 231 & 76 & 8394.8 & 8.44 \\
FK745823 & BDS-14 & 231 & 76 & 8272.7 & 8.96 \\
FK725211 & BDS-15 & 231 & 76 & 8317.8 & 8.93 \\
\hline
\end{tabular}

\footnotetext{
${ }^{a}$ Length of cDNA, stop codon is included; ${ }^{b}$ Length (No. of amino acids) of the deduced polypeptide;
}

${ }^{\mathrm{c}}$ Molecular weight of the deduced polypeptide in Dalton; ${ }^{\mathrm{d}}$ Isoelectric point of the deduced polypeptide. 
BDS cDNA sequences exhibited high sequence identity in the ORFs, as well as in the $3^{\prime}$ and $5^{\prime}$ untranslated regions (UTRs) (Figure 1). In particular, BDS-1 displayed the highest sequence similarity with BDS-3, BDS-5, BDS-7, BDS-12 and BDS-15 (99\% identity). Additionally, BDS-1 showed identity ranging from $98 \%$ to $96 \%$ with BDS-4, BDS-6, BDS-8, BDS-9, BDS-10, BDS-11 and BDS-13, while the lowest similarity was achieved comparing BDS-1 with BDS-14 (94\% identity). A comparison of cDNAs encoding BDS-9 and BDS-11 showed that the two putative toxins are identical, except for a shorter 5' UTR in the BDS-9 EST sequence; consequently, BDS-9 and BDS-11 were considered one (and indicated as BDS-11) for all subsequent computational and experimental analyses.

BDS-7, BDS-8 and BDS-10 EST sequences each contain a single nucleotide insertion upstream of the start codon (ATG); moreover, a few nucleotide substitutions were found in the same regions.

Compared with the other cDNAs, BDS-6, BDS-8, BDS-10, and BDS-11 contained insertion elements (12-bp insertion in BDS-6 and BDS-11, 4-bp insertion in BDS-10 and 3-bp insertion in BDS-8) mapping downstream of the start codon and located at a position between 176 and 187 in the alignment. A deletion of the BDS-10 ORF abrogates the conserved stop codon (TAG), causing the continuation of protein synthesis until the downstream stop codon (TGA) is encountered. Consequently, BDS-10 has the longest ORF of all the ESTs.

A total of 473 sequenced base pairs were aligned, including the coding and non-coding regions, and after trimming, nucleotide sequence analyses were conducted on the region between positions 68 and 473 in the alignment. The sequence analysis revealed that 18 sites showed alignment gaps; 53 sites were variable, and 19 of these sites were parsimony-informative. Considering the regions between positions 68 and 307 of the alignment 42 sites were variable, and 18 were parsimony-informative; while, between positions 68 and 325 of the alignment, 43 sites were variable and 18 were parsimony informative. The protein sequence alignment contains 85 positions (Figure 2). Considering all the alignments, 24 amino acid sites were variable, and 9 of these sites are parsimony-informative.

Figure 2. Protein sequence alignment of BDS peptides from $A$. viridis performed with ClustalW2. Below the sequences, asterisks mark cysteine residues located at the same positions.

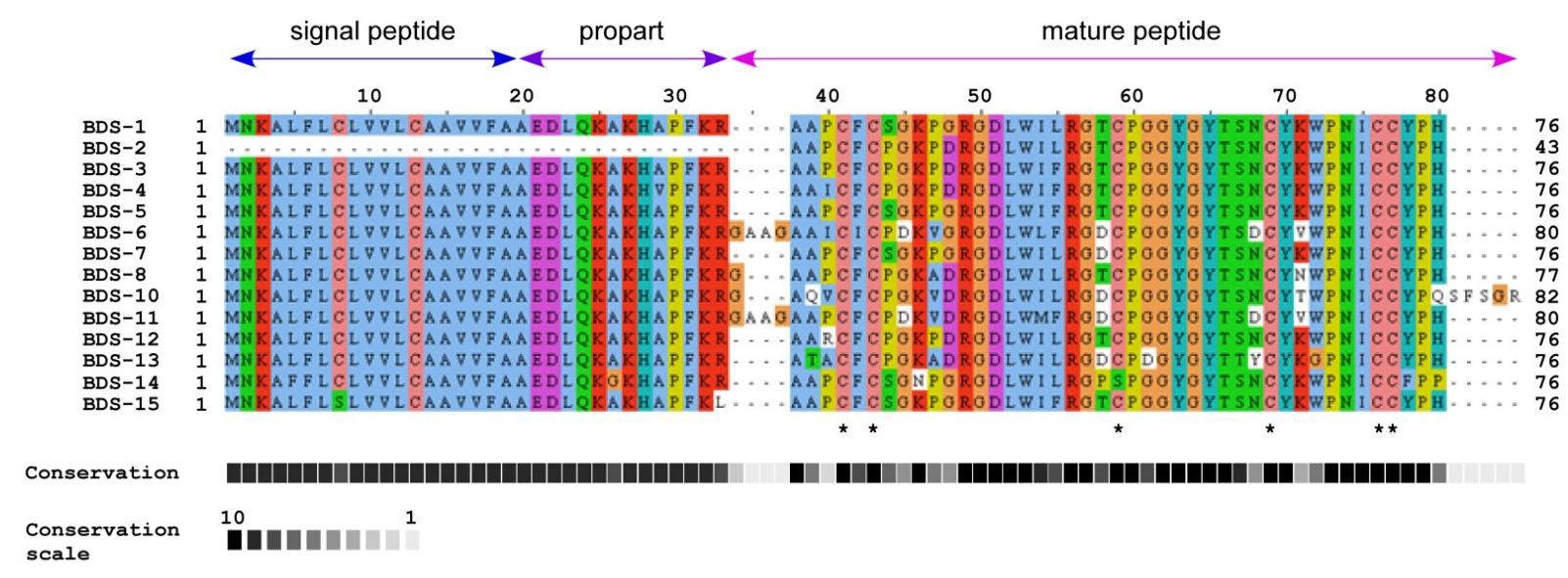

To obtain further information about the evolution of BDS sequences, the number of nucleotide substitutions per site $\left(K_{\mathrm{N}}\right)$ in the UTR regions and the number of nucleotide substitutions per synonymous site $\left(K_{\mathrm{S}}\right)$ and non-synonymous site $\left(K_{\mathrm{A}}\right)$ in the protein-coding region were calculated for the BDS 
cDNA sequences. Based on the neutral theory of molecular evolution [21], the $K_{\mathrm{A}}$ value never exceeds the $K_{\mathrm{S}}$ value. If $K_{\mathrm{A}}$ were to exceed $K_{\mathrm{S}}, K_{\mathrm{A}} / K_{\mathrm{S}}>1$ would result and serve as evidence that positive selection had acted to change the protein-coding region. The $K_{\mathrm{N}} / K_{\mathrm{S}}$ ratios were less than 1 , while the $K_{\mathrm{A}} / K_{\mathrm{S}}$ ratios calculated for all pairwise comparisons ranged from 0.98 to 4.4 , with an average value of 1.81. This pattern is indicative of strong positive selection for amino acid replacements because it implies that the rate of non-synonymous changes exceeds that which can be explained solely by a neutral substitution rate. These emerging features reflect the evolutionary mechanisms described previously for some toxins affecting voltage-gated sodium channels from sea anemones [2,3], isozymes of snake venom [22,23], and toxins from scorpions [24], which have evolved in an adaptive manner under positive Darwinian selection ("accelerated evolution"). Hence, selection promotes the fixation of non-synonymous substitutions and accelerates the diversification of related sequences.

\subsection{Comparison of BDS Amino Acid Sequences}

The characteristics of the analysed BDS protein sequences are summarised in Table 1. The predicted proteins have an estimated molecular mass ranging from 8317.8 (BDS-15) to $9041.5 \mathrm{Da}$ (BDS-10) and a theoretical pI between 8.14 and 9.02 .

The deduced BDS neurotoxins are synthesised as inactive precursors with a putative N-terminal leader peptide (residues 1-19), a propart of 14 residues, a cleavage site for proteolytic activation, and the mature toxins (Figure 2). The propart is composed primarily of polar and negatively charged amino acids and is cleaved upon toxin maturation. In addition, the propart has been proposed to have a role in intracellular sorting and delivery to target the toxin to the nematocyst $[25,26]$. However, the presence of a propart is not limited to neurotoxin peptides because it has also been identified in cytolysins from Actineria villosa [27]. Although the BDS peptide sequences are highly similar, BDS-6, BDS-8, BDS-10 and BDS-11 contain one or four additional residues at the $N$-termini of their mature peptides. BDS-10 also contains an extra five residues at the $C$-terminus due to the absence of the otherwise highly conserved stop codon (TAG).

BDS-15 differs from all other BDS peptides because it lacks the highly conserved dyad $\mathrm{K}^{32} \mathrm{R}^{33}$, and Cys is substituted with Ser at position 8 in the leader peptide. This result is anomalous if compared with previous reports on sea anemone toxins containing a tandem Lys-Arg at the C-terminal end of the propart $[18,25,26,28]$. In this context, BDS-15 appears to resemble other proteins, including SNTX [29], Galectin-3 [30], Hk2a [31] and AvTX-20 [27] toxins. Consequently, a different secretory mechanism, without the involvement of the endoplasmic reticulum or Golgi apparatus, which was hypothesised for such proteins [32], might also be extended to BDS-15. The processing required for toxin activation may not be essential for cellular activity, as demonstrated previously for the pertussis toxin [33]. Nevertheless, the effective functionality of the putative BDS-15 toxin remains unaddressed.

\subsection{Sequence Similarity with Other Peptide Toxins}

A basic local alignment search tool (BLASTp) analysis indicated that all BDS toxins share moderate to high similarity with other sea anemone toxins active on voltage-dependent $\mathrm{K}^{+}$channels. Mature BDS peptides displayed identity ranging from $40 \%$ to $46 \%$ with the Am II toxin of Antheopsis maculata, U-AITX-Bg1, and Bgr3 peptides from Bunodosoma granuliferum. Significant similarity 
32 amino acid Am-II precursor from A. maculata. With the exception of BDS-15, the subtilisin-like protease cleavage site (Lys-Arg or Arg-Arg) is conserved among all the sequences; additionally, the signal peptides and proparts are related.

Table 2. Kv channel toxins from sea anemones, accession numbers and related references.

\begin{tabular}{|c|c|c|c|}
\hline Species & Toxin & GenBank accession number & References \\
\hline \multirow[t]{4}{*}{ Bunodosoma cangicum } & Bcg III 29.21 & P86464 & Zaharenko et al. 2008 [34] \\
\hline & Bcg III 25.52 & P86463 & \\
\hline & Bcg III 28.78 & P86462 & \\
\hline & Bcg III 31.16 & P86461 & \\
\hline \multirow[t]{5}{*}{ Bunodosoma granuliferum } & U-AITX-Bg1a & CCC86602 & Rodriguez et al. 2012 [35] \\
\hline & U-AITX-Bg1b & CCC86603 & \\
\hline & U-AITX-Bg1c & $\mathrm{CCC} 86604$ & \\
\hline & U-AITX-Bgr3c & CCC86605 & \\
\hline & U-AITX-Bgr3d & CCC86606 & \\
\hline \multirow[t]{2}{*}{ Bunodosoma caissarum } & $\mathrm{BcIV}$ & P86470 & Oliveira et al. 2006 [36] \\
\hline & $\mathrm{BcV}$ & P84919 & Zaharenko, A.Z. [37] \\
\hline Antheopsis maculata & Am II & P69930 & Honma et al. 2005 [11] \\
\hline \multirow[t]{4}{*}{ Anthopleura elegantissima } & APETx1 & P61541 & Diochot et al. 2003 [10] \\
\hline & APETx 2 & P61542 & \\
\hline & APETx3 & B3EWF9 & Peigneur, S. [38] \\
\hline & U-AITX-Aella & FG392547* & Richier et al. 2008 [39] \\
\hline
\end{tabular}

* FG392547 refers to an EST derived by Richier et al. (2008) [39]; The deduced amino acid was reported by Rodriguez et al. 2012 [35] as U-AITX-Ael1; however, no accession number has been associated.

\subsection{Phylogenetic Analysis}

To investigate the evolutionary relationships among the toxins affecting $\mathrm{K}^{+}$channels, a phylogenetic tree was constructed using the 14 mature BDS toxins and the mature protein sequences of 16 sea anemone neurotoxins (Figure 4).

Phylogenetic analysis displayed the presence of two well-defined clades; the first clade represents the 14 BDS toxins from $A$. viridis, the Am-II peptide from A. maculata, the U-AITX-Bgr3d from Bunodosoma granulifera and Bcg III 25.52 from B. cangicum. The second clade groups the BDS-like peptides, as inferred from Pfam and InterPro annotations, from A. elegantissima (U-AITX-Ael1a, APETx1, APETx2 and APETx3), B. cangicum (Bcg III 28.78, Bcg III 29.21, and Bcg III 31.16), B. caissarum (BcIV and $\mathrm{BcV}$ ), and B. granulifera (U-AITX-Bg1a, U-AITX-Bg1b, U-AITX-Bg1c and U-AITX-Bgr3c).

\subsection{Homology Modelling}

To establish conserved structural motifs, the 3D structures of the BDS toxins were predicted by homology modelling using BDS-I (PDB ID 1bds) as the template, and the generated models were validated by assessing a Ramachandran plot (Figure 5). The results of these analyses showed that the percentage of residues in the favoured/allowed region ranged from $90 \%$ to $95 \%$ among all the predicted models. 
Figure 4. Neighbour-Joining (NJ) phylogenetic tree based on 14 BDS amino acid sequences and other cnidarian $\mathrm{Kv}$ channel toxins from GenBank using the $p$-distance model. Toxin BmKK2 (Q95NK7) from Mesobuthus martensii was used as outgroup. Internal branches were assessed using 1000 bootstrap replications. Branches corresponding to positions reproduced in less than $40 \%$ bootstrap replicates are collapsed.

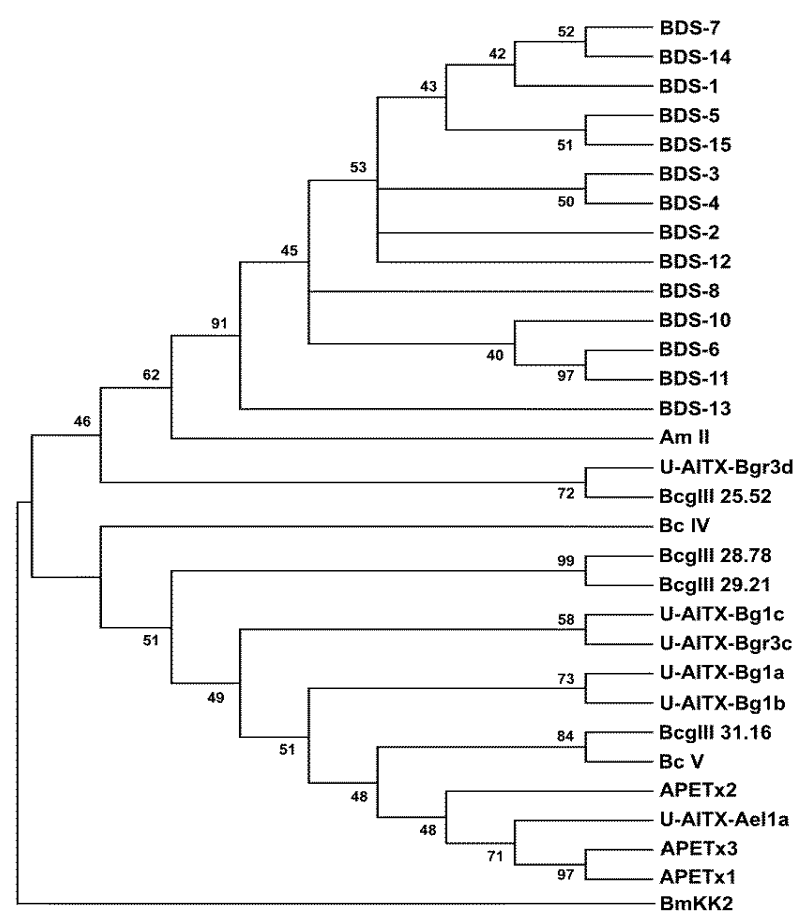

Figure 5. Representative Ramachandran maps of the predicted 3D model of two BDS peptides (BDS-3 on the left; BDS-6 on the right). Each amino acid residue is shown as a symbol in a graph of $\varphi v s . \psi$. For further details, refer to the keys beneath the maps.

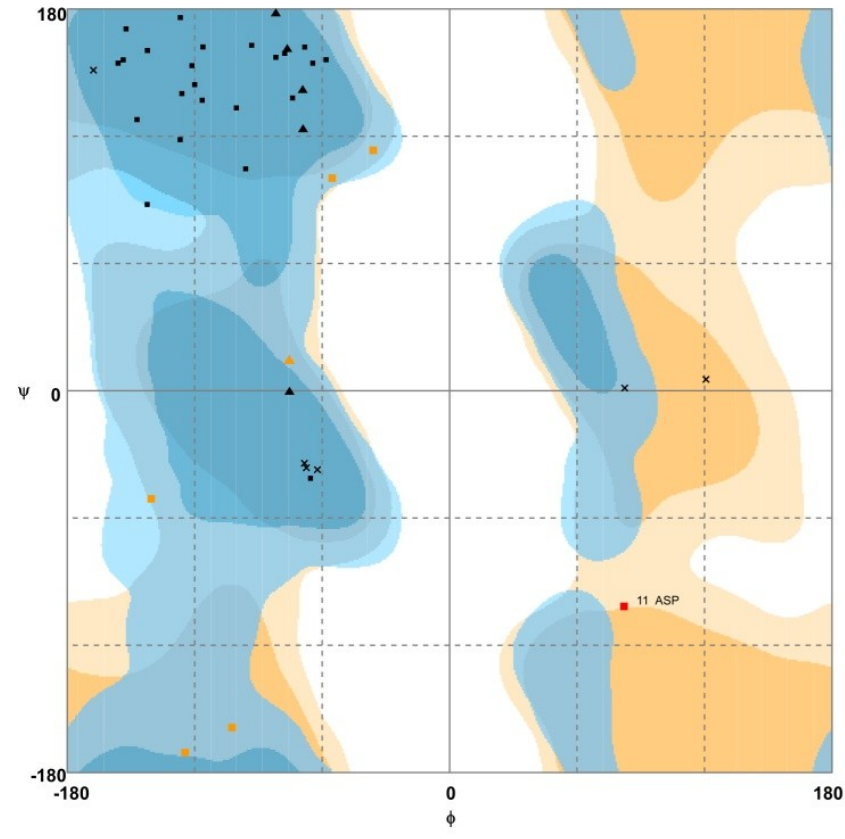

Glycine Favoured

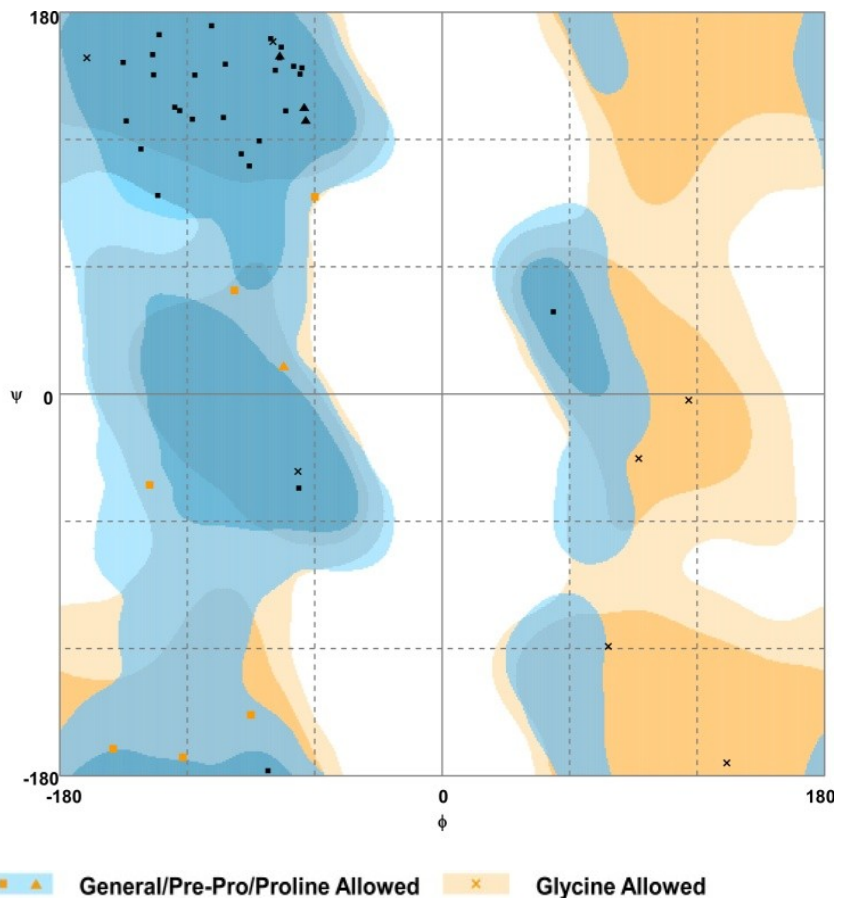


Modelling analysis predicted $\beta$-strand configurations for the mature BDS toxins (Figure 6), as previously described for the mature BDS-1 and BDS-2 toxins [40,41], and $\alpha$-helical structures in the leader peptides and proparts (data not shown).

Figure 6. Homology modelling of BDS toxins. The models were built based on the NMR structure of the mature form of BDS-1. (A) BDS-1 surface in an electrostatic potential model; $(\mathbf{B}, \mathbf{C})$ Structural elements of BDS-1 and BDS-2; (D) Structure predictions of the BDS toxins. The colour transition from blue to red represents the transition from the $N$ - to $C$-terminus.

A

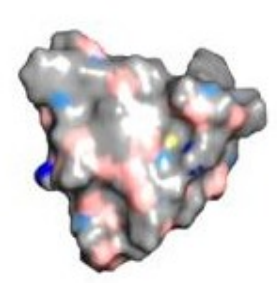

D

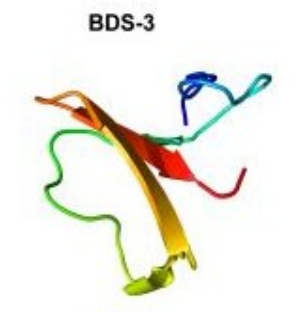

BDS- 6

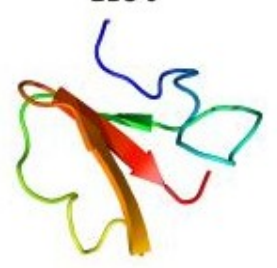

BDS-10

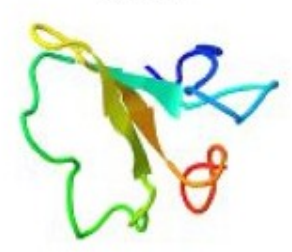

BDS-13

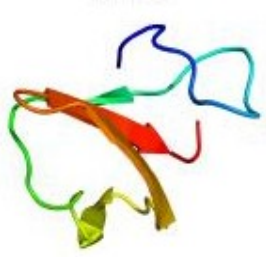

B

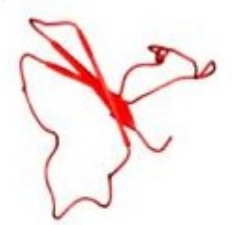

BDS-4
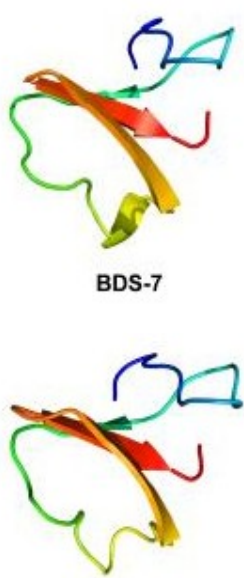

BDS-11

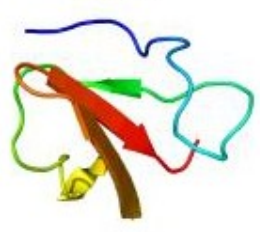

BDS-14

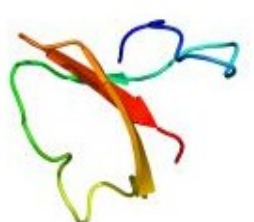

C

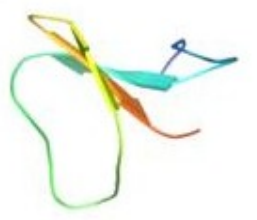

BDS-5

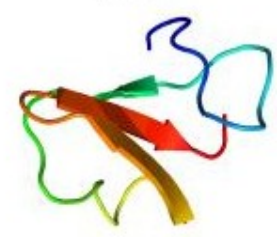

BDS-8

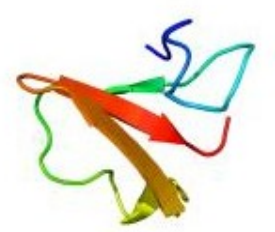

BDS-12

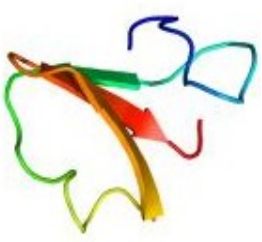

BDS-15

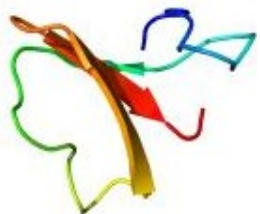

The mature toxins are usually organised in a triple-stranded antiparallel $\beta$-sheet. Loops connecting the strands of the antiparallel $\beta$-sheet and an additional antiparallel $\beta$-strand located downstream of the cleavage/maturation site are also present. Despite the observed residue substitutions and the insertion of the tripeptide $\mathrm{A}^{36} \mathrm{GA}^{38}$ into BDS- 6 and BDS-11 as well as the pentapeptide $\mathrm{S}^{78} \mathrm{FSGR}^{82}$ into BDS-10, a comparison of BDS structures reveals that the toxins possess grossly similar 3D structures. However, the orientation of the triple-stranded $\beta$-sheet seems to be different and change among the toxins. Thus, 
such changes might influence the activity and specificity of the neurotoxins constituting the BDS arsenal of $A$. viridis.

\subsection{Tissue-Specific Gene Expression Pattern}

Real-time polymerase chain reaction (RT-PCR) represents a robust technique with which to study the expression profile of a specific gene. However, due to the low variability among BDS cDNA sequences, it was not possible to design a suitable primer set to selectively amplify the sequence variants. Instead, sequencing randomly selected clones from cDNA libraries is a useful alternative to investigate genes and gene expression patterns in a tissue of interest [42].

To analyse the tissue distribution and expression profiles of BDS toxins at the transcriptional level, total RNA was isolated from ectodermal- and endodermal-derived tissues. To monitor contamination of ectodermal or endodermal extracts, the transcripts for an isoform of carbonic anhydrase 2 (CA2-M) and hydroxymethylglutaryl-CoA reductase (HMG-CoA reductase) [43] were reverse transcribed and amplified in all samples as endoderm and ectoderm markers, respectively. CA2-M and HMG-R amplicons were usually detected after 20 cycles in endodermal and ectodermal tissues, respectively, and limited cross-contamination was evident after 30 cycles of amplification. Additionally, CA2-M and HMG-R transcripts were co-expressed in the pharynx and muscles (data not shown).

To avoid biases that could result in the loss of certain species of BDS transcripts, tissue-specific cDNA libraries enriched for BDS transcripts were generated. A limited number of amplification cycles were performed to minimise the amount of contaminating cDNA sequences that could impair the results and to ensure that high target concentrations did not reach saturation.

Tissue-specific cDNA clones were screened by colony PCR, and 200 independent clones per tissue were sequenced. BDS transcripts were detected in ectodermal tissue from the distal and proximal tentacles (the tentacle's base), body wall, and oral disk, with different relative abundances (Figure 7). In total, transcripts of ten BDS representatives were identified, while BDS-8, BDS-14 and BDS-15 were not retrieved. In contrast, no BDS transcripts were detected in the pharynx, gonads, and muscle tissues, although these tissues contain endodermal and ectodermal tissues. This result indicates that these transcripts are extremely underrepresented in our cDNA libraries, either due to low expression levels or a very restricted expression pattern. The ectodermal cells constituting the external epithelial layer of tentacles, body wall and oral disk exerts several functions including defence against predators and capturing and killing prey. The ectodermal cells of the pharynx, gonads, and muscles participate in different physiological functions, which include digestion, gamete development and movement processes. Thus, the presence of cells responsible for the active transcription and production of BDS neurotoxins is not required in these tissue areas. However, the limited number of cycles performed for the preparation of the cDNA libraries may have contributed to a slight underrepresentation.

The relative abundance of a clone was considered to be a proxy for transcriptional activation, and as shown in Figure 7, the levels of BDS-1 transcript in our ectodermal cDNA libraries were the highest in all the analysed tissues with a relative abundance ranging from $21 \%$ to $34 \%$. According to the literature [20], BDS-1 is the most represented component of the family in A. viridis. Levels of expression from high to moderate were also shown for BDS-3, BDS-4, BDS-5 and BDS-6; conversely, the number of clones for BDS-7, BDS-10, BDS-11, BDS-12 and BDS-13 cDNAs was less abundant. 
To validate the results, the relative abundances of BDS transcripts were compared to the number of ESTs encoding the BDS toxins in the $A$. viridis database, and a total of 49 EST sequences were collected. The cDNAs encoding BDS-3, BDS-4, BDS-5 and BDS-6 were well represented due to the presence of five or six EST sequences each; meanwhile, ESTs encoding BDS-7, BDS-10 and BDS-11 showed lower representation. The cDNAs encoding BDS-8, BDS-14 and BDS-15 were the least abundant (one EST encoding each toxin). This result may explain why no clones encoding these putative toxins were retrieved after sequencing our cDNA libraries.

Figure 7. Relative frequencies of BDS clones in ectodermal tissues of $A$. viridis. Variations in the number of clones in the library were considered a measure of gene expression.
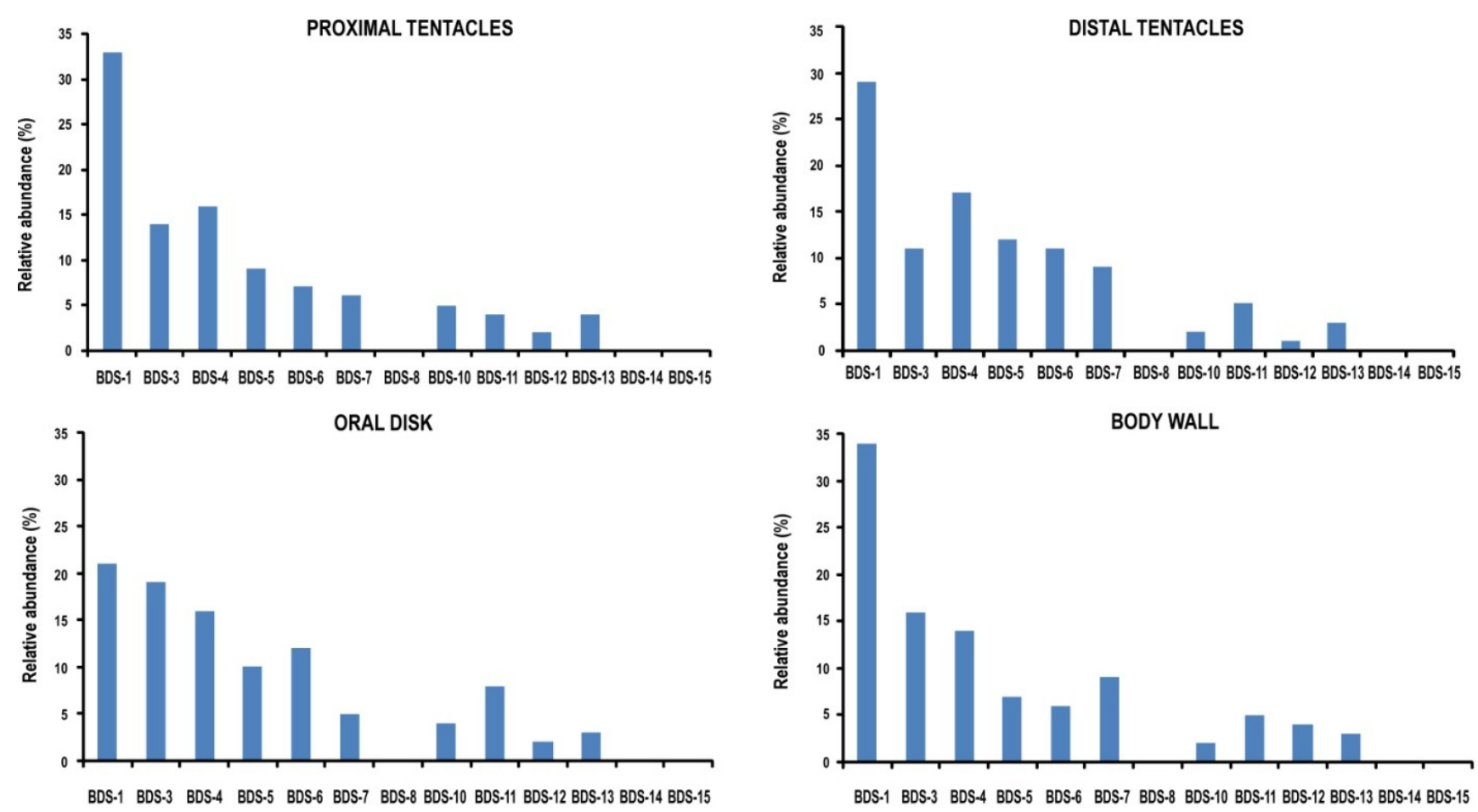

\section{Experimental Section}

\subsection{GenBank Accession Numbers}

The BDS sequences were obtained from the $A$. viridis EST database [19] at the National Centre for Biotechnology Information (NCBI) under the following accession numbers: FK728690 (BDS-1), FK744472 (BDS-3), FK722457 (BDS-4), FK720902 (BDS-5), FK754940 (BDS-6), FK736435 (BDS-7), FK723172 (BDS-8), FK721972 (BDS-9), FK725608 (BDS-10), FK740326 (BDS-11), FK736010 (BDS-12), FK752236 (BDS-13) and FK745823 (BDS-14) [20]. The BDS-15 sequence, accession number FK725211, was extracted from the $A$. viridis EST database using the TBLASTN search tool.

\subsection{Sequence and Phylogenetic Analyses}

The nucleotide and deduced amino acid sequences of BDS cDNAs were compared with other known sequences, including the BDS-II toxin (P59084), using the BLAST algorithm and the ExPASy 
Bioinformatics Resource Portal. Nucleotide polymorphisms and substitution rates among the BDS ESTs were determined using DNAsp software [44] and Molecular Evolution Genetics Analyses (MEGA) software version 5.0 [45].

We computed the number of conserved, variable, and parsimony-informative sites. The number of nucleotide substitutions per site $\left(K_{\mathrm{N}}\right)$ in the untranslated regions (UTRs) and the number of nucleotide substitutions per synonymous site $\left(K_{\mathrm{S}}\right)$ and non-synonymous site $\left(K_{\mathrm{A}}\right)$ in the protein-coding region were computed for all the pairwise comparisons of BDS sequences according to the method of Nei and Gojobori [46]. $K_{\mathrm{A}} / K_{\mathrm{S}}$ ratios were used to evaluate the selective forces acting on these proteins.

Signal peptides, functional sites and domains in the predicted amino acid sequences were predicted by the Simple Modular Architecture Research Tool (SMART) program, the InterPro database, the Pfam database, the PROSITE program, and the Eukaryotic Linear Motif resource (ELM) for Functional Sites in Proteins. Multiple sequence alignments were performed using the ClustalW 2.0 program at the European Bioinformatics Institute. Phylogenetic and molecular evolutionary analyses were conducted on the amino acid sequences of the mature BDS toxins and 16 cnidarian Kv channel toxins (Table 2) using the Neighbour-Joining (NJ) method in MEGA 5.0. Evolutionary distances were estimated using the $p$-distance model, and alignment gaps were deleted prior to calculation. Internal branch support was assessed using 1000 bootstrap replications. A phylogenetic tree was determined using the BDS toxins and other cnidarian neurotoxin sequences recovered in GenBank and reported in Table 2. The $\mathrm{K}^{+}$channel blocker BmKK2 toxin (GenBank Accession Number: Q95NK7) from the venom of chinese scorpion Mesobuthus martensii was used as outgroup.

\subsection{Homology Modelling}

BDS 3D structures were reconstructed by homology modelling via the Protein Homology/analogY Recognition Engine 2.0 (Phyre 2) software [47], using the intensive modelling mode. Candidate structures for homology modelling were selected according to pairwise alignment and cysteine distribution. The NMR 3D solution structure of BDS-1 (PDB code: 1bds) was used as a template, and homology models were built for all of the sets of proteins. Validation of the structural protein models was performed by assessing the Ramachandran plots. Cycles of clash minimisation were also performed for the refinement of structures.

\subsection{RNA Extraction and First-Strand cDNA Synthesis}

Specimens of $A$. viridis were collected from the Capo Granitola coast (Torretta Granitola, Trapani, Italy) in the south of Sicily and maintained in a $200 \mathrm{~L}$ aerated seawater tank. Tentacles, oral disks, and body walls were cut lengthwise and scraped to separate the endodermal cell layer and the film of mesogloea from the ectodermal cells. The small size of the pharynx, muscles and gonads prevented the separation of ectodermal from endodermal tissue; consequently, RNA was isolated from both ectodermal and endodermal layers. Tissues were frozen in liquid nitrogen and ground into a fine powder using a tissue disruptor. The powder was dissolved in Trizol reagent (Invitrogen Corporation, Carlsbad, CA, USA), and further RNA purification steps were performed according to the manufacturer's instructions. RNA concentrations and quality were verified by spectrophotometry (optical density (OD) at $260 \mathrm{~nm}$ ), while RNA integrity was checked using a 1.5\% agarose gel. The 
RNA was stored at $-80^{\circ} \mathrm{C}$ for future use. The extracted RNA $(2 \mu \mathrm{g})$ was treated with RNA qualified 1 (RQ1) RNase-Free DNase (Promega, Madison, WI, USA) to remove any residual genomic DNA contamination, and DNase I was inactivated by adding $25 \mathrm{mM}$ EDTA. First-strand cDNA was synthesised from $2 \mu \mathrm{g}$ DNase I-treated total RNA samples using oligo(dT) $)_{18}$ and Superscript III (Invitrogen Corporation), following the manufacturer's instructions. The cDNA mixture was stored at $-20{ }^{\circ} \mathrm{C}$ until needed.

\subsection{Tissue-Specific BDS cDNA Library Construction}

RNA quality and cross-contamination were assessed by performing RT-PCR reactions using CA2-M and HMG-R specific primers (Table 3), and the expected bands were obtained.

Table 3. Oligonucleotide primers used in this study.

\begin{tabular}{ccc}
\hline Primers & Sequences $\left(\mathbf{5}^{\prime}-\mathbf{3}^{\prime}\right)$ & Amplicon size (bp) \\
\hline BDS-F & GAAAATGAACAAAGCTCTTTCC & \multirow{2}{*}{$278-290$} \\
BDS-R & GATCGGACTGATGTTACTGG & \\
CA2-m-F & CTTTGGCGGCATTTCACTTG & \multirow{2}{*}{129} \\
CA2-m-R & GTGATTGGTTGGAGCCATCG & \\
HMG-F & AGTATGTGAAGCCATAGTGC & \multirow{2}{*}{311} \\
HMG-R & TAGTACCACCACCAACAGTC & \\
\hline
\end{tabular}

The cDNAs derived from the ectodermal and endodermal tissues were separately subjected to PCR reactions using a pair of primers (BDS-F and BDS-R) specifically chosen to ensure the amplification of all the BDS sequence variants in the EST database.

PCR reactions were performed using AmpliTaq Gold DNA polymerase (Applied Biosystems, Forster City, CA, USA) with the following conditions: Pre-denaturation at $94{ }^{\circ} \mathrm{C}$ for $4 \mathrm{~min}, 25$ cycles of $94{ }^{\circ} \mathrm{C}$ for $30 \mathrm{~s}, 52{ }^{\circ} \mathrm{C}$ for $30 \mathrm{~s}$, and $72{ }^{\circ} \mathrm{C}$ for $30 \mathrm{~s}$, followed by elongation at $72{ }^{\circ} \mathrm{C}$ for $8 \mathrm{~min}$.

Amplified products that were representative of BDS sequence variants in each studied tissue were analysed using a 1.4\% agarose gel and subcloned into the pGEM-T Easy vector (Promega).

The resulting tissue-specific libraries were transformed into the Escherichia coli strain DH10B (Promega) and seeded onto $150 \mathrm{~mm}$ Luria-Bertani-Agar plates containing $100 \mu \mathrm{g} / \mathrm{mL}$ of ampicillin. Library screening was performed by colony PCR using the BDS-F and BDS-R primers.

From each library, 200 recombinant clones were picked and replicated, and the plasmid DNA was purified using a QIAprep Spin Miniprep Kit (QIAGEN, Tokio, Japan) and sequenced using T7 and SP6 primers on an ABI-3730 Genetic Analyser (Applied Biosystems). The relative abundance of a clone was computed by dividing the number of a specific BDS clone with the total number of sequenced clones per library.

\section{Conclusions}

Sea anemones are a well-known source of neurotoxins acting upon voltage-gated sodium and potassium ion channels [48]. A. viridis, the Mediterranean species of sea anemone, is unable to retract and is exposed to attack from predators, such as Nudibranchia. Moreover, A. viridis captures food actively and feeds on a wide spectrum of prey, including crustaceans and molluscs [49]. Thus, the 
chemical arsenal of $A$. viridis represents the preferred strategy to survive in such a habitat and the components of the produced neurotoxin cocktail are very copious [2,50].

In the present study, comparative analysis of BDS cDNA sequences allowed us to hypothesise that mechanisms of accelerated evolution occurred in the protein-coding region of BDS peptides. Additionally, although the 3D structure of BDS toxins remains grossly unchanged, the orientation of the triple stranded $\beta$-sheet appears to be modified among the models. Such diversity and variations may be responsible for the activity on $\mathrm{K}^{+}$channels containing $\mathrm{Kv} 3$ subunits and also for the inactivation of Nav1.7 and Nav1.3 channels exerted by preparations of extracted BDS toxins [16].

Because the capture and killing of prey as well as mechanisms of defence and protection in sea anemones are closely related to toxin production, the presence of multiple variants generated by accelerated evolution could provide some benefit. The variety of putative BDS toxins produced by A. viridis could reflect the high environmental adaptability of the species. Previous studies showed that the evolution of toxin diversity is linked to the diet and ecology of a species [51,52]. Thus, similar mechanisms appear to have affected the diversity of BDS toxins, equipping the sea anemone with important systems for survival.

Herein, the spatial expression profile of the putative BDS cDNA sequences was also examined. A recent report has demonstrated that Type I neurotoxins from Nematostella vectensis, A. elegantissima and $A$. viridis are confined to nematocytes and ectodermal gland cells [53]. Despite phylogeny and different channel specificity, gene expression studies revealed that BDS transcripts were co-expressed in ectodermal tissue. Further studies focused on the identification of BDS toxin-synthesising cells will be helpful to gain further insight on mechanisms of toxin production and delivery.

In addition to their natural role in sea anemones, BDS toxins were considered of scientific interest in Central Nervous System (CNS) studies since their first characterisation. Several studies reported the use of such antagonists as a pharmacological tool to assign functional roles to channels in CNS neurons [54-56]. Because the Kv3 potassium channels are essential in CNS physiological activity and Kv3.4 subunits were shown to be involved in responses to chronic hypoxia [57] in Parkinson's [55] and Alzheimer's [58] diseases, selective targeting of the aforementioned subunits will have therapeutic utility in CNS disorders.

Recombinant DNA technology associated with functional bioassays could allow a specific BDS toxin to be produced in heterologous expression systems. This technology could lead to the development of new peptides with restricted activity as biopharmaceuticals for the treatment of neuronal diseases.

\section{Acknowledgments}

We thank Fabrizio Gianguzza for allowing us to carry out most experiments in his laboratory, Carmelo Bennici for the dissection of sea anemone tissues and the anonymous reviewers whose comments helped us to improve and clarify this manuscript.

\section{Conflicts of Interest}

The authors declare no conflict of interest. 


\section{References}

1. Ruppert, E.E.; Barnes, R.D. The Cnidarians. In Invertebrate Zoology, 6th ed.; Saunders College Publishing: Philadelphia, PA, USA, 1994; pp. 103-154.

2. Moran, Y.; Weinberger, H.; Sullivan, J.C.; Reitzel, A.M.; Finnerty, J.R.; Gurevitz, M. Concerted evolution of sea anemone neurotoxin genes is revealed through analysis of the Nematostella vectensis genome. Mol. Biol. Evol. 2008, 25, 737-747.

3. Moran, Y.; Weinberger, H.; Reitzel, A.M.; Sullivan, J.C.; Kahn, R.; Gordon, D.; Finnerty, J.R.; Gurevitz, M. Intron retention as a posttranscriptional regulatory mechanism of neurotoxin expression at early life stages of the starlet anemone Nematostella vectensis. J. Mol. Biol. 2008, 380, 437-443.

4. Moran, Y.; Weinberger, H.; Lazarus, N.; Gur, M.; Kahn, R.; Gordon, D.; Gurevitz, M. Fusion and retrotransposition events in the evolution of the sea anemone Anemonia viridis neurotoxin genes. J. Mol. Evol. 2009, 69, 115-124.

5. Venn, A.A.; Tambutte, E.; Lotto, S.; Zoccola, D.; Allemand, D.; Tambutte, S. Imaging intracellular pH in a reef coral and symbiotic anemone. Proc. Natl. Acad. Sci. USA 2009, 106, 16574-16579.

6. Castaneda, O.; Harvey, A.L. Discovery and characterization of cnidarian peptide toxins that affect neuronal potassium ion channels. Toxicon 2009, 54, 1119-1124.

7. Frazão, B.; Vasconcelos, V.; Antunes, A. Sea anemone (Cnidaria, Anthozoa, Actiniaria) toxins: An overview. Mar. Drugs 2012, 10, 1812-1851.

8. Honma, T.; Shiomi, K. Peptide toxins in sea anemones: Structural and functional aspects. Mar. Biotechnol. 2006, 8, 1-10.

9. Honma, T.; Kawahata, S.; Ishida, M.; Nagai, H.; Nagashima, Y.; Shiomi, K. Novel peptide toxins from the sea anemone Stichodactyla haddoni. Peptides 2008, 29, 536-544.

10. Diochot, S.; Loret, E.; Bruhn, T.; Beress, L.; Lazdunski, M. APETx1, a new toxin from the sea anemone Anthopleura elegantissima, blocks voltage-gated human ether-a-go-go-related gene potassium channels. Mol. Pharmacol. 2003, 64, 59-69.

11. Honma, T.; Hasegawa, Y.; Ishida, M.; Nagai, H.; Nagashima, Y.; Shiomi, K. Isolation and molecular cloning of novel peptide toxins from the sea anemone Antheopsis maculata. Toxicon 2005, 45, 33-41.

12. Béress, L.; Doppelfeld, I.S.; Etschenberg, E.; Graf, E.; Henschen, A.; Zwick, J. Polypeptides, Methods of Production and Their Uses as Antihypertensives. German Patent DE3324689A1, 17 January 1985.

13. Diochot, S.; Schweitz, H.; Beress, L.; Lazdunski, M. Sea anemone peptides with a specific blocking activity against the fast inactivating potassium channel Kv3.4. J. Biol. Chem. 1998, 273, 6744-6749.

14. Yeung, S.Y.M.; Thompson, D.; Wang, Z.; Fedida, D.; Robertson, B. Modulation of Kv3 subfamily potassium currents by the sea anemone toxin BDS: Significance for CNS and biophysical studies. J. Neurosci. 2005, 38, 8735-8745.

15. Abbott, G.W.; Butler, M.H.; Bendahhou, S.; Dalakas, M.C.; Ptacek, L.J.; Goldstein, S.A. MiRP2 forms potassium channels in skeletal muscle with $\mathrm{Kv} 3.4$ and is associated with periodic paralysis. Cell 2001, 104, 217-231. 
16. Liu, P.; Jo, S.; Bean, B.P. Modulation of neuronal sodium channels by the sea anemone peptide BDS-I. J. Neurophysiol. 2012, 107, 3155-3167.

17. Pannaccione, A.; Boscia, F.; Scorziello, A.; Adornetto, A.; Castaldo, P.; Sirabella, R.; Taglialatela, M.; di Renzo, G.F.; Annunziato, L. Upregulation and increased activity of KV3.4 channels and their accessory subunit MinK-related peptide 2 induced by amyloid peptide are involved in apoptotic neuronal death. Mol. Pharmacol. 2007, 272, 665-673.

18. Urbarova, I.; Karlsen, B.O.; Okkenhaug, S.; Seternes, O.M.; Johansen, S.D.; Emblem, A. Digital marine bioprospecting: Mining new neurotoxin drug candidates from the transcriptomes of cold-water sea anemones. Mar. Drugs 2012, 10, 2265-2279.

19. Sabourault, C.; Ganot, P.; Deleury, E.; Allemand, D.; Furla, P. Comprehensive EST analysis of the symbiotic sea anemone, Anemonia viridis. BMC Genomics 2009, 10, 333.

20. Kozlov, S.; Grishin, E. The mining of toxin-like polypeptides from EST database by single residue distribution analysis. BMC Genomics 2011, 12, 88.

21. Kimura, M. The Neutral Theory of Molecular Evolution; Cambridge University Press: Cambridge, UK, 1983.

22. Ogawa, T.; Oda, N.; Nakashima, K.; Sasaki, H.; Hattori, M.; Sakaki, Y.; Kihara, H.; Ohno, M. Unusually high conservation of untranslated sequences in cDNAs for Trimeresurus flavoviridis phospholipase A2 isozymes. Proc. Natl. Acad. Sci. USA 1992, 89, 8557-8561.

23. Deshimaru, M.; Ogawa, T.; Nakashima, K.; Nobuhisa, I.; Chijiwa, T.; Shimohigashi, Y.; Fukumaki, Y.; Niwa, M.; Yamashina, I.; Hattori, S.; et al. Accelerated evolution of crotalinae snake venom gland serine proteases. FEBS Lett. 1996, 397, 83-88.

24. Zhu, S.; Bosmans, F.; Tytgat, J. Adaptive evolution of scorpion sodium channel toxins. J. Mol. Evol. 2004, 58, 145-153.

25. Anderluh, G.; Podlesek, Z.; Macek, P. A common motif in proparts of Cnidarian toxins and nematocyst collagens and its putative role. Biochim. Biophys. Acta 2000, 1476, 372-376.

26. Anderluh, G.; Macek, P. Cytolytic peptide and protein toxins from sea anemones (Anthozoa: Actiniaria). Toxicon 2002, 40, 111-124.

27. Nagai, H.; Oshiro, N.; Takuwa-Kuroda, K.; Iwanaga, S.; Nozaki, M.; Nakajima, T. A new polypeptide toxin from the nematocyst venom of an Okinawan sea anemone Phyllodiscus semoni (Japanese name "unbachi-isoginchaku"). Biosci. Biotechnol. Biochem. 2002, 66, 2621-2625.

28. Spagnuolo, A.; Zanetti, L.; Cariello, L.; Piccoli, R. Isolation and characterization of two genes encoding calitoxins, neurotoxic peptides from Calliactis parasittica (Cnidaria). Gene 1994, 138, 187-191.

29. Ghadessy, F.J.; Chen, D.; Kini, R.M.; Chung, M.C.; Jeyaseelan, K.; Khoo, H.E.; Yuen, R. Stonustoxin is a novel lethal factor from stonefish (Synanceja horrida) venom. cDNA cloning and characterization. J. Biol. Chem. 1996, 271, 25575-25581.

30. Menon, R.P.; Hughes, R.C. Determinants in the N-terminal domains of galectin-3 for secretion by a novel pathway circumventing the endoplasmic reticulum-Golgi complex. Eur. J. Biochem. 1999, 264, 569-576.

31. Liu, W.H.; Wang, L.; Wang, Y.L.; Peng, L.S.; Wu, W.Y.; Peng, W.L.; Jiang, X.Y.; Tu, H.B.; Chen, H.P.; Ou-Yang, P.; et al. Cloning and characterization of a novel neurotoxin from the sea anemone Anthopleura sp. Toxicon 2003, 41, 793-801. 
32. Muesch, A.; Hartmann, E.; Rohde, K.; Rubartelli, A.; Sitia, R.; Rapoport, T.A. A novel pathway for secretory proteins? Trends Biochem. Sci. 1990, 15, 86-88.

33. Carbonetti, N.H.; Mays, R.M.; Artamonova, G.V.; Plaut, R.D.; Worthington, Z.E. Proteolytic cleavage of pertussis toxin S1 subunit is not essential for its activity in mammalian cells. BMC Microbiol. 2005, 5, 7.

34. Zaharenko, A.J.; Ferreira, W.A., Jr.; Oliveira, J.S.; Richardson, M.; Pimenta, D.C.; Konno, K.; Portaro, F.C.V.; Freitas, J.C. Proteomics of the neurotoxic fraction from the sea anemone Bunodosoma cangicum venom: Novel peptides belonging to new classes of toxins. Comp. Biochem. Physiol. Part D 2008, 3, 219-225.

35. Rodríguez, A.A.; Cassoli, J.S.; Sa, F.; Dong, Z.Q.; de Freitas, J.C.; Pimenta, A.M.; de Lima, M.E.; Konno, K.; Lee, S.M.; Garateix, A.; et al. Peptide fingerprinting of the neurotoxic fractions isolated from the secretions of sea anemones Stichodactyla helianthus and Bunodosoma granulifera. New members of the APETx-like family identified by a 454 pyrosequencing approach. Peptides 2012 , $34,26-38$.

36. Oliveira, J.S.; Zaharenko, A.J.; Ferreira, W.A., Jr.; Konno, K.; Shida, C.S.; Richardson, M.; Lúcio, A.D.; Beirão, P.S.; de Freitas, J.C. BcIV, a new paralyzing peptide obtained from the venom of the sea anemone Bunodosoma caissarum. A comparison with the $\mathrm{Na}^{+}$channel toxin BcIII. Biochim. Biophys. Acta 2006, 1764, 1592-1600.

37. Zaharenko, A.Z. University of São Paulo, São Paulo, SP, Brazil. Unpublished work, 2010.

38. Peigneur, S. University of Leuven, Leuven, Belgium. Unpublished work, 2012.

39. Richier, S.; Rodriguez-Lanetty, M.; Schnitzler, C.E.; Weis, V.M. Response of the symbiotic cnidarian Anthopleura elegantissima transcriptome to temperature and UV increase. Comp. Biochem. Physiol. Part D 2008, 3, 283-289.

40. Driscoll, P.C.; Clore, G.M.; Beress, L.; Gronenborn, A.M. A proton nuclear magnetic resonance study of the antihypertensive and antiviral protein BDS-I from the sea anemone Anemonia sulcata: Sequential and stereospecific resonance assignment and secondary structure. Biochemistry 1989, $28,2178-2187$.

41. Driscoll, P.C.; Gronenborn, A.M.; Beress, L.; Clore, G.M. Determination of the three-dimensional solution structure of the antihypertensive and antiviral protein BDS-I from the sea anemone Anemonia sulcata: A study using nuclear magnetic resonance and hybrid distance geometry-dynamical simulated annealing. Biochemistry 1989, 28, 2188-2198.

42. Venter, J.C.; Levy, S.; Stockwell, T.; Remington, K.; Halpern, A. Massive parallelism, randomness and genomic advances. Nat. Genet. 2003, 33, 219-227.

43. Ganot, P.; Moya, A.; Magnone, V.; Allemand, D.; Furla, P.; Sabourault, C. Adaptations to endosymbiosis in a cnidarian-dinoflagellate association: Differential gene expression and specific gene duplications. PLoS Genet. 2011, 7, e1002187.

44. Librado, P.; Rozas, J. DNAsp v5: A software for comprehensive analysis of DNA polymorphism data. Bioinformatics 2009, 25, 1451-1452.

45. Tamura, K.; Peterson, D.; Peterson, N.; Stecher, G.; Nei, M.; Kumar, S. MEGA 5: Molecular evolutionary genetics analysis using maximum likelihood, evolutionary distance, and maximum parsimony method. Mol. Biol. Evol. 2011, 28, 2731-2739. 
46. Nei, M.; Gojobori, T. Simple methods for estimating the numbers of synonymous and non synonymous nucleotide substitutions. Mol. Biol. Evol. 1986, 3, 418-426.

47. Kelley, L.A.; Sternberg, M.J.E. Protein structure prediction on the web: A case study using the Phyre server. Nat. Protoc. 2009, 4, 363-371.

48. Yamaguchi, Y.; Hasegawa, Y.; Honma, T.; Nagashima, Y.; Shiomi, K. Screening and cDNA cloning of Kv1 potassium channel toxins in sea anemones. Mar. Drugs 2010, 8, 2893-2905.

49. Chintiroglou, C.; Koukouras, A. The feeding habits of three Mediterranean sea anemone species, Anemonia viridis (Forskal), Actinia equina (Linnaeus) and Cereus pedunculatus (Pennant). Helgol. Meeresunters. 1992, 46, 53-68.

50. Moran, Y.; Gordon, D.; Gurevitz, M. Sea anemone toxins affecting voltage-gated sodium channels: Molecular and evolutionary features. Toxicon 2009, 54, 1089-1101.

51. Barlow, A.; Pook, C.E.; Harrison, R.A.; Wuster, W. Coevolution of diet and prey-specific venom activity supports the role of selection in snake venom evolution. Proc. R. Soc. B 2009, 276, 2443-2449.

52. Fry, B.G.; Vidal, N.; van der Weerd, L.; Kochva, E.; Renjifo, C. Evolution and diversification of the toxicofera reptile venom system. J. Proteomics 2009, 72, 127-136.

53. Moran, Y.; Genikhovich, G.; Gordon, D.; Wienkoop, S.; Zenkert, C.; Ozbek, S.; Technau, U.; Gurevitz, M. Neurotoxin localization to ectodermal gland cells uncovers an alternative mechanism of venom delivery in sea anemones. Proc. Biol. Sci. B 2012, 279, 1351-1358.

54. Chabbert, C.; Chambard, J.M.; Sans, A.; Desmadryl, G. Three types of depolarization-activated potassium currents in acutely isolated mouse vestibular neurons. J. Neurophysiol. 2001, 85, 1017-1026.

55. Baranauskas, G.; Tkatch, T.; Nagata, K.; Yeh, J.Z.; Surmeier, D.J. Kv3.4 subunits enhance the repolarizing efficiency of Kv3.1 channels in fast-spiking neurons. Nat. Neurosci. 2003, 6, 258-266.

56. Shevchenko, T.; Teruyama, R.; Armstrong, W.E. High-threshold, Kv3-like potassium currents in magnocellular neurosecretory neurons and their role in spike repolarization. J. Neurophysiol. 2004, 92, 3043-3055.

57. Kaab, S.; Miguel-Velado, E.; Lopez-Lopez, J.R.; Perez-Garcia, M.T. Down regulation of Kv3.4 channels by chronic hypoxia increases acute oxygen sensitivity in rabbit carotid body. J. Physiol. (Lond.) 2005, 566, 395-408.

58. Angulo, E.; Noe, V.; Casado, V.; Mallol, J.; Gomez-Isla, T.; Lluis, C.; Ferrer, I.; Ciudad, C.J.; Franco, R. Up-regulation of the Kv3.4 potassium channel subunit in early stages of Alzheimer's disease. J. Neurochem. 2004, 91, 547-557.

(C) 2013 by the authors; licensee MDPI, Basel, Switzerland. This article is an open access article distributed under the terms and conditions of the Creative Commons Attribution license (http://creativecommons.org/licenses/by/3.0/). 